\title{
Tdp-43 cryptic exons are highly variable between cell types
}

\author{
Yun Ha Jeong ${ }^{1,5 \dagger}$, Jonathan P. Ling ${ }^{1 \dagger}$, Sophie Z. Lin ${ }^{1 \dagger}$, Aneesh N. Donde ${ }^{1,2}$, Kerstin E. Braunstein ${ }^{1}$, Elisa Majounie ${ }^{4,6}$, \\ Bryan J. Traynor ${ }^{3,4}$, Katherine D. LaClair ${ }^{1}$, Thomas E. Lloyd ${ }^{2,3}$ and Philip C. Wong 1,2*
}

\begin{abstract}
Background: TDP-43 proteinopathy is a prominent pathological feature that occurs in a number of human diseases including amyotrophic lateral sclerosis (ALS), frontotemporal dementia (FTD), and inclusion body myositis (IBM). Our recent finding that TDP-43 represses nonconserved cryptic exons led us to ask whether cell type-specific cryptic exons could exist to impact unique molecular pathways in brain or muscle.
\end{abstract}

Methods: In the present work, we investigated TDP-43's function in various mouse tissues to model disease pathogenesis. We generated mice to conditionally delete TDP-43 in excitatory neurons or skeletal myocytes and identified the cell type-specific cryptic exons associated with TDP-43 loss of function.

Results: Comparative analysis of nonconserved cryptic exons in various mouse cell types revealed that only some cryptic exons were common amongst stem cells, neurons, and myocytes; the majority of these nonconserved cryptic exons were cell type-specific.

Conclusions: Our results suggest that in human disease, TDP-43 loss of function may impair cell type-specific pathways.

Keywords: TDP-43 -Nonconserved cryptic exons, Bioinformatics, Amyotrophic lateral sclerosis, Frontotemporal dementia, Inclusion body myositis

\section{Background}

Recent genetic evidence has established the linkage between the neurological disorders amyotrophic lateral sclerosis (ALS) and frontotemporal dementia (FTD) [1-5]. The key pathological feature that is shared between ALS and FTD is the cytoplasmic aggregation and nuclear clearance of an RNA binding protein called transactive response DNA binding protein $43 \mathrm{kDa}$ (TDP-43, TARDBP) [6]. Since the discovery of TDP-43, a number of other human diseases have also been characterized with TDP-43 pathology [7-12]. Of particular interest, however, is the pathogenesis of inclusion body myositis (IBM), which is believed to be primarily myogenic rather than neurogenic $[13,14]$. To understand the mechanisms of

\footnotetext{
* Correspondence: wong@jhmi.edu

${ }^{\dagger}$ Equal contributors

'Departments of Pathology, Johns Hopkins University School of Medicine, Baltimore, MD 21205, USA

${ }^{2}$ Departments of Neuroscience, Johns Hopkins University School of Medicine, Baltimore, MD 21205, USA

Full list of author information is available at the end of the article
}

disease pathogenesis that will inform appropriate therapeutic strategies, it will be critical to determine whether the pathways affected by TDP-43 proteinopathy differ between neurons and myocytes.

We have recently found that TDP-43 plays a major role in repressing nonconserved cryptic exons [15]. These cryptic exons are regions of the genome that are normally skipped by the spliceosome due to the presence of adjacent UG microsatellite repeats, the consensus binding site of TDP-43. When TDP- 43 function is lost, these cryptic exons become activated and often lead to nonsense-mediated decay (NMD) of the associated mRNA. In our previous report [15], we utilized an in vitro inducible stem cell model of TDP-43 deletion. However, we have yet to establish the cell type-specific cryptic exons that arise in vivo. Here, we generated conditional Tdp- 43 knockout mice to specifically delete Tdp-43 in excitatory neurons and skeletal myocytes. We found that Tdp- 43 cryptic exons are highly variable between cell types and that many distinct pathways are 
altered-novel findings that have mechanistic and therapeutic implications for human diseases exhibiting TDP43 proteinopathy.

\section{Methods}

\section{Mouse breeding strategy}

We crossbred our conditional Tardbp knockout mice $\left(\operatorname{Tardbp}^{\mathrm{F} /+}\right)$ with CamKIIa-Cre transgenic mice to obtain a cohort of CamKIIa-Cre;Tardbp ${ }^{\mathrm{F} /+}$ mice which were subsequently crossbred to $\operatorname{Tardbp}^{\mathrm{F} /+}$ mice to generate the final cohort: CamKIIa-Cre;Tardbp ${ }^{+/+}$, CamKIIa-Cre;Tardbp ${ }^{\mathrm{F} /+}$ and CamKIIa-Cre;Tardbp ${ }^{\mathrm{F} / \mathrm{F}}$ mice. A similar strategy was applied when crossbreeding the $M L C$-Cre driver line to $\operatorname{Tardbp}^{\mathrm{F} /+}$ mice. All mouse experiments were approved by the Johns Hopkins University Animal Care and Use Committee.

\section{Histology and immunohistochemistry}

For the CamKIIa-Cre line, wildtype and floxed mice were anaesthetized and perfused with $4 \%$ paraformaldehyde. Brains were embedded into paraffin, cut into $10 \mu \mathrm{m}$ sections and stained according to standard protocols. For the $\mathrm{MLC}$-Cre line, wildtype and floxed mice were anaesthetized and sacrificed by decapitation. Muscle tissue was then rapidly dissected and flash frozen in liquid nitrogen cooled isopentane. Frozen cryosections were cut at $10 \mu \mathrm{m}$ thickness and stained according to standard protocols. Immunoreactivity was visualized using the Vectastain $\mathrm{ABC}$ Kit and diaminobenzidine peroxidase substrate (Vector Laboratories). Images were obtained using Olyumpus BX53 microscope.

\section{Immunoblot analysis}

For the CamKIIa-Cre line, wildtype and floxed mice were anaesthetized and sacrificed by decapitation. Brain tissue was then rapidly dissected and manually homogenized in RIPA buffer (Sigma) containing an EDTA-free protease inhibitor cocktail (Thermo Scientific). For the $M L C$-Cre line, wildtype and floxed mice were also anaesthetized and sacrificed by decapitation. Muscle tissue was snap frozen in isopentane cooled with liquid nitrogen, manually ground into a powder, and then homogenized in RIPA buffer with protease inhibitor cocktail. Protein concentration was determined using the BCA assay (Pierce). Proteins were resolved using the NuPAGE 4-12\% Bis-Tris Gel (Novex) with NuPAGE MES SDS Running Buffer (Novex), and transferred to PVDF membrane (Millipore) with NuPAGE Transfer Buffer (Invitrogen).

The following antibodies were used for protein blots, immunofluorescence, and immunohistochemical analyses: rabbit anti-TDP-43 (Proteintech 10782-2-AP and 128921-AP), anti-NeuN monoclonal antibody (Chemicon), antiGAPDH monoclonal antibody (Sigma), Alexa Fluor 488- conjugated Donkey anti-Guinea Pig IgG $(\mathrm{H}+\mathrm{L})$ antibody (Jackson ImmunoResearch), Alexa Fluor 594- and 647conjugated Donkey anti-goat and anti-rabbit IgG $(\mathrm{H}+\mathrm{L})$ antibodies (Life Tech.).

\section{RNA extraction, RNA-seq analysis}

Total RNA was extracted from hippocampi of 3 month old female CamKIIa-Cre;Tardbp ${ }^{\mathrm{F} / \mathrm{F}}$ (neuronal knockout) and littermate control mice (CamKIIa-Cre;Tardbp ${ }^{+/+}$) using TRIzol (Life Tech.) and RNeasy Mini kits (Qiagen). Total RNA from 2 month old male $M L C$-Cre;Tardbp ${ }^{\mathrm{F} / \mathrm{F}}$ (skeletal muscle knockout) and littermate control mice $\left(M L C-C r e ; \operatorname{Tardbp}^{+/+}\right)$was also extracted in a similar manner. For the CamKIIa-Cre line, 3 control brains and 3 knockout brains were analyzed and all mice were female. For the $M L C$-Cre line, 2 control quadriceps and 2 knockout quadriceps were analyzed and all mice were male. 100-bp paired end RNA-seq libraries were generated using Illumina Tru-seq kits and then sequenced on an Illumina HiSeq 2000. For RT-PCR analysis, total RNA was isolated using RNeasy Mini Kit (Qiagen). cDNA was synthetized using RevertAid First Strand cDNA Synthesis Kit (Thermo Scientific) with random primers. RNA-seq analysis was performed using HISAT [16] and Cufflinks [17] software suites and visualized on the UCSC Genome Browser [18]. Cryptic exons were identified as previously described [14]. To identify common pathways between species, gene ontology analysis was performed on cryptic exon targets using manual annotation of genes with known functions in combination with the bioinformatics resource DAVID v6.7 [19].

\section{RT-PCR primers}

\begin{tabular}{lll}
\hline Primer & Sequence & Tissue \\
\hline Ap3b2-Forward & AGCCAGAATATGGCCACGAC & Neuron \\
Ap3b2-Reverse & CACTATGATGGGCACACGGA & Neuron \\
Camk1g-Forward & CTGGCCAAGATCACAGACTGG & Neuron \\
Camk1g-Reverse & CTGTGTAGACACCACGCTCT & Neuron \\
Sh3bgr-Forward & GGAGCAGAGGCTTGGATCAC & Muscle \\
Sh3bgr-Reverse & AAAGCCCACCACTTCTTGCT & Muscle \\
Tns1-Forward & CCTGGTCTATCAGCACTCCG & Muscle \\
Tns1-Reverse & GGGCTCCCGATTCGTTCAT & Muscle \\
\hline
\end{tabular}

\section{Results}

Selective deletion of Tdp-43 in mouse excitatory neurons and skeletal myocytes

To identify the cryptic exons repressed by Tdp- 43 in neurons and myocytes, we utilized the Cre recombinase system to conditionally delete Tdp-43. Mice harboring floxed Tardbp knockout alleles [20] were crossbred with 
either CaMKII $\alpha$-Cre [21] or MLC-Cre [22] driver lines (Fig. 1a). The promoter of the calcium/calmodulindependent protein kinase II alpha subunit (CaMKII $\alpha)$ drives expression primarily in the excitatory neurons of the cortex and hippocampus whereas the promoter of the myosin light chain $1 / 3$ locus $(M L C)$ drives expression in type II fasttwitch skeletal muscle fibers. Efficient deletion of Tdp- 43 can be detected by immunoblot in brain (Fig. 1b) and skeletal muscle (Fig. 1c); residual Tdp-43 in F/F mice reflects the presence of other cell types that do not express $\mathrm{CaM}$ KII $\alpha$-Cre or MLC-Cre. Neuron specific deletion of Tdp- 43 was confirmed by immunofluorescence staining of hippocampal sections (Fig. 1d); deletion of Tdp-43 in myocytes was also verified by immunohistochemistry (Fig. 1e).

Identification of cryptic exons associated with Tdp-43 loss of function in neurons and myocytes

To identify the cryptic exons of mouse neurons, RNAsequencing (RNA-seq) analysis was performed using RNA extracted from hippocampi of 3 month old CaM$K I I \alpha-C r e ; T_{a r d b p}{ }^{F / F}$ mice and controls. Similar to our in vitro stem cell culture model of Tdp-43 deletion [15], we also found cryptic exons in the brains of CaMKII $\alpha$-Cre;$\operatorname{Tardbp}^{F / F}$ knockout mice (Fig. 2a). Neuron-specific cryptic exons were still flanked by UG microsatellite repeats (Fig. 2b) and could be classified as standard cryptic exons, transcriptional start sites, exon extensions or premature polyadenylation sites (Additional file 1: Table S4, Additional file 1: Figure S1). Previously published CLIP data was also able to confirm the presence of a direct interaction with Tdp-43 (Additional file 1: Figure S2) [23]. Finally, to further validate our RNA-seq data, RT-PCR analysis was able to confirm the presence of cryptic exons in the genes Camk1g and Ap3b2. Longer PCR products, indicating cryptic exon inclusion, were detected in CaMKII $\alpha$-Cre;Tardbp ${ }^{F / F}$ knockout but not control mice (Fig. 2c-e).

To determine whether cryptic exons of mouse myocytes would differ from those found in stem cells and neurons, we also performed RNA-seq analysis on quadriceps muscle from $M L C$-Cre;Tardbp ${ }^{F / F}$ knockout mice and controls. Indeed, numerous muscle-specific cryptic

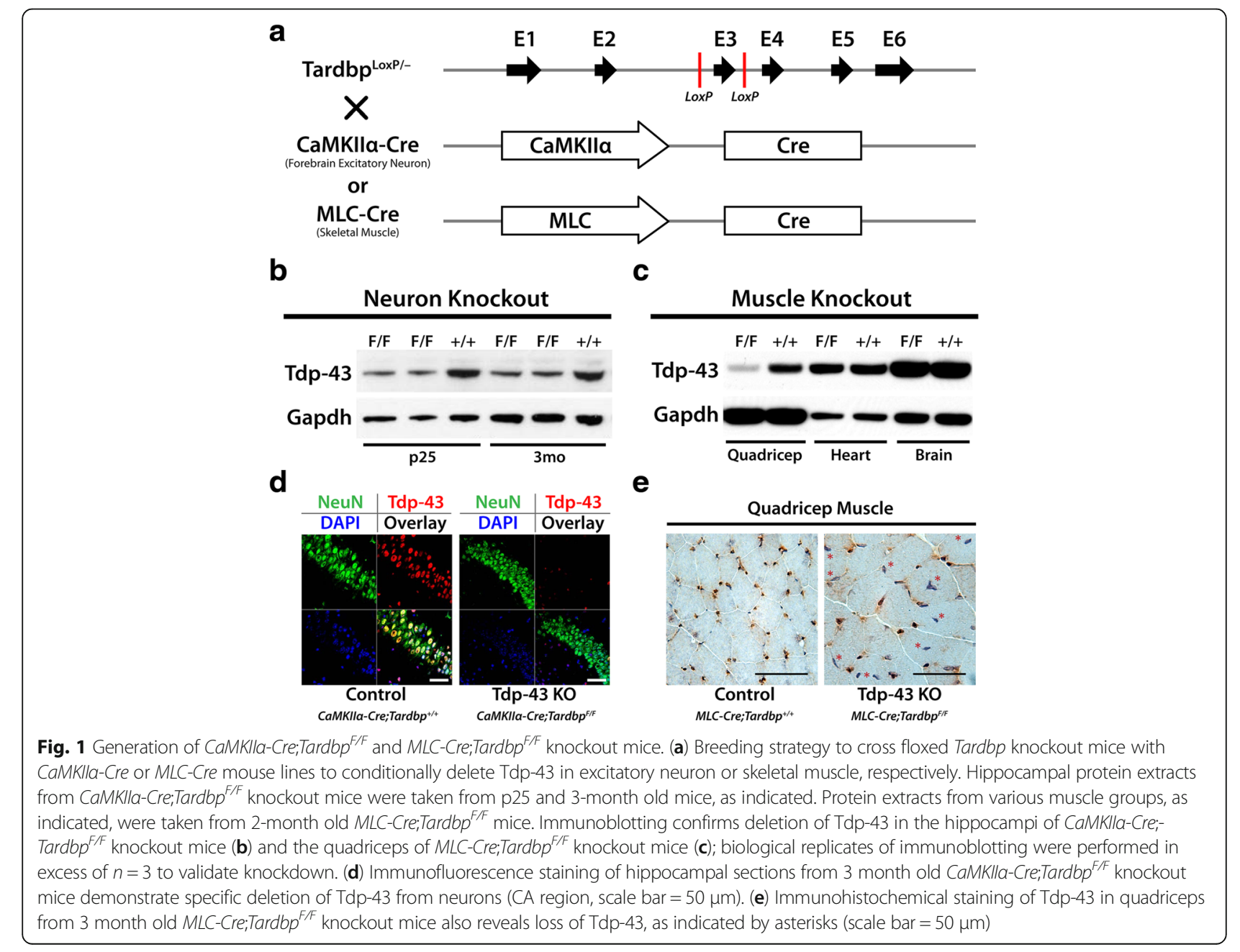




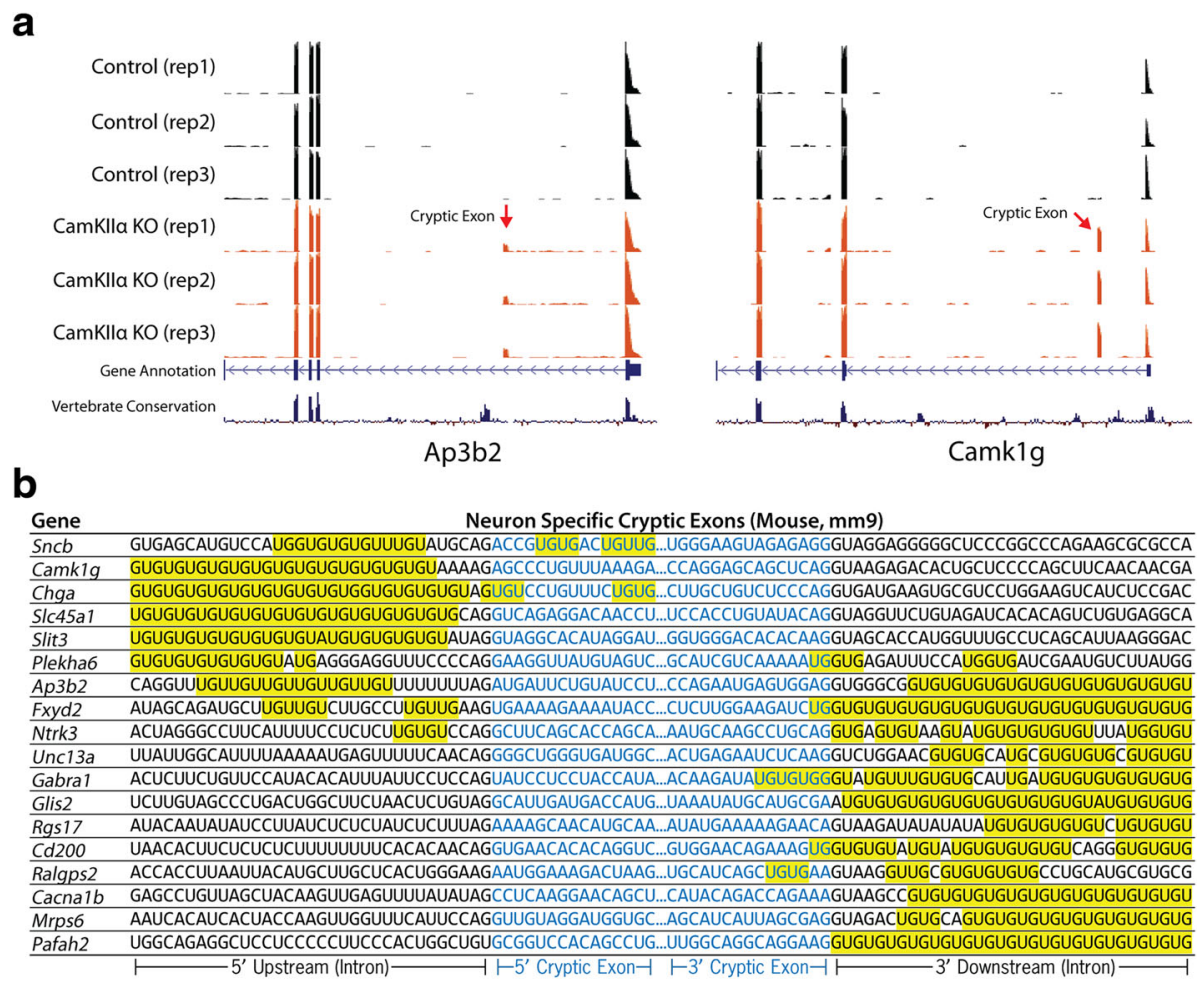

C

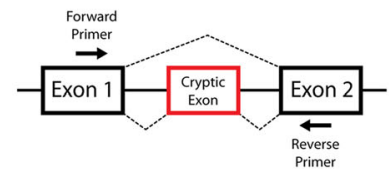

d

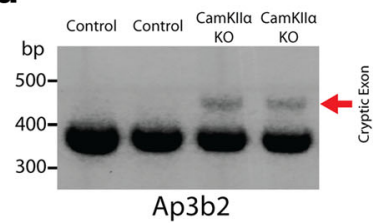

e

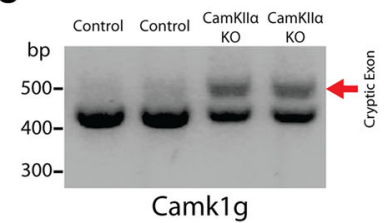

Fig. 2 Neuron-specific cryptic exons (CaMKIla-Cre;Tardbp F/F knockout mice). (a) Visual examples of neuron-specific cryptic exons (Ap3b2, Camk1g). (b) Neuron-specific cryptic exons are flanked by UG repeats that are present upstream, downstream or within the cryptic exon sequence itself. (c to e) RT-PCR validation of cryptic exons (red arrows) in RNA extracted from hippocampi of 3 month old CaMKlla-Cre;Tardbp ${ }^{F / F}$ mice. Refer to Additional file 2 for a complete list of cryptic exons

exons could be identified (Fig. 3a). Furthermore, myocyte-specific cryptic exons were also flanked by UG microsatellite repeats (Fig. 3b); the presence of cryptic exons was confirmed by RT-PCR as shown for two genes, Sh3bgr and Tns1 (Fig. 3c).

\section{Unique Tdp-43 cryptic exons occur in stem cells, neurons,} and myocytes

Having identified two new sets of cryptic exons belonging to mouse neurons and myocytes, we compared these sites with the cryptic exons previously identified in mouse stem cells [15]. Interestingly, only 66/221 ( 30\%) total cryptic exons showed any overlap between at least two cell types and only $32 / 221(\sim 14 \%)$ were common among all three cell types (Fig. 4a). Although the ratios varied, the majority of cryptic exons were unique to each individual cell type $(155 / 221 ; \sim 70 \%)$. When normalized to the total number of cryptic exons in stem cells (74), neurons (109) and myocytes (136), the number of cell type-specific cryptic exons was lower in stem cells (18; $\sim 24 \%)$ as compared to neurons $(58 ; \sim 53 \%)$ and myocytes (79; $58 \%)$. These results indicate that a large proportion of Tdp-43's cryptic exons are cell type-specific (Additional file 1: Table S1 and S2).

Differential levels of cryptic exon incorporation, however, increase the complexity of these cryptic exon datasets. While certain cryptic exons, such as those in Synj2bp and Adnp2, can be observed at high levels in all three cell types (Fig. 4b), it is more common to see differential usage of cryptic exons amongst stem cells, neurons, and myocytes despite abundant transcription of the associated mRNA (Fig. 4c-g). For example, the cryptic exon in Ube $2 d 1$ is highly incorporated in stem cells, moderately incorporated in myocytes, and absent in neurons (Fig. 4c). Conversely, the cryptic exon in Rrp36 is high in neurons but low in stem cells and myocytes 


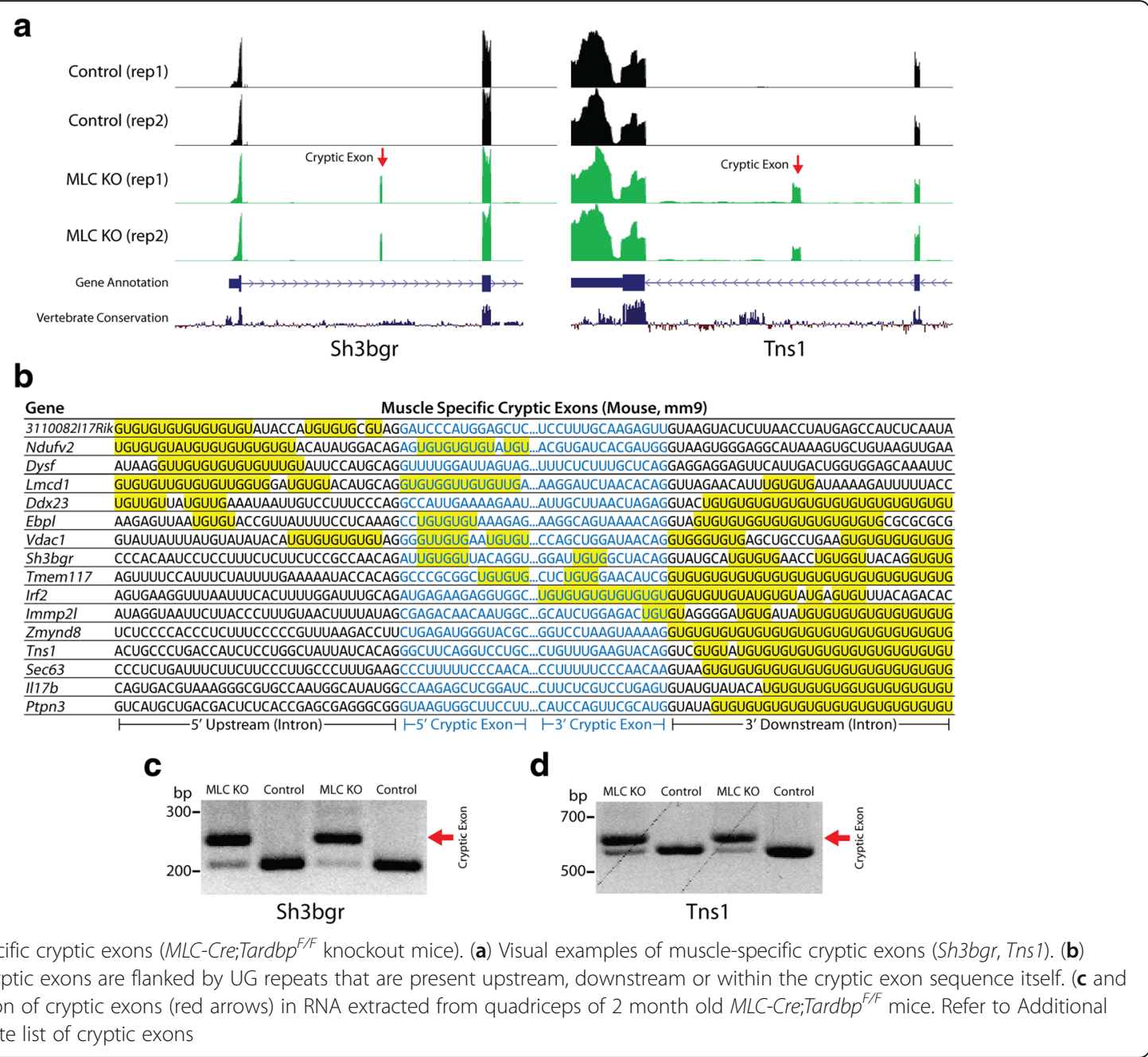

(Fig. 4d). Thus, it appears that the activation of a cryptic exon within a specific cell type depends not only upon transcription of the associated mRNA, but also the local splicing factor environment present within the cell (Additional file 1: Figure S3).

\section{Comparative analysis of genes affected by cryptic exon disruption}

We have previously shown that Tdp-43's nonconserved cryptic exons could disrupt gene function in cultured stem cells [15]. Similarly, while some neuron and myocyte cryptic exons reside in the 5' or 3' untranslated regions ( $19 \%)$ with no clear effect on transcript levels, the majority of cryptic exons disrupt normal protein translation by introducing premature stop codons that lead to nonsense mediated decay (Additional file 1: Figure S4) or early termination of the mRNA transcript ( 63\%). Of these disrupted genes, numerous critical pathways are affected, ranging from mitochondrial function and protein regulation to transcriptional control and genome stability (Table 1). These findings demonstrate that cell type- specific pathways are altered when Tdp-43 function is lost and suggest that unique molecular pathways could differentially impact ALS-FTD and IBM.

\section{Common pathways affected by Tdp-43 loss of function}

Although many cryptic exons are predicted to induce nonsense mediated decay, their impact on mRNA and protein levels depends upon the frequency of cryptic exon incorporation. Across stem cells, neurons and myocytes, a broad group of genes are affected by Tdp- 43 loss of function (Table 1). Many pathways are affected, from mitochondrial function and cell growth to transcription and genomic regulation, offering a possible explanation for the observed cell death associated with Tdp-43 deletion [24-28]; CaMKII $\alpha-C r e ; T a r d b p^{F / F}$ exhibit significant cortical atrophy at 8 months of age [12] while $M L C$-Cre;Tardbp ${ }^{F / F}$ mice reach endstage by $4-5$ months. Several other genes that are disrupted by cryptic exons also reflect previously reported observations: Drosha is involved in miRNA biogenesis [29], Tecpr1 is 


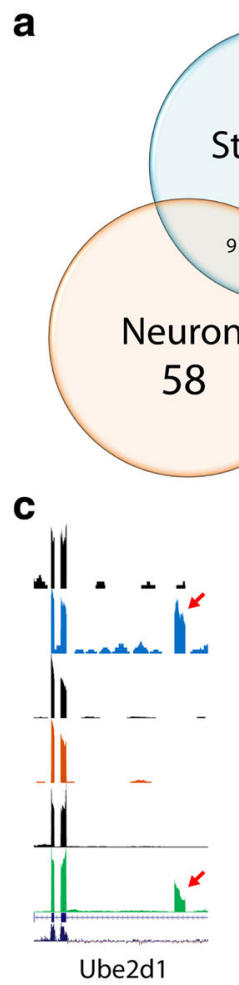

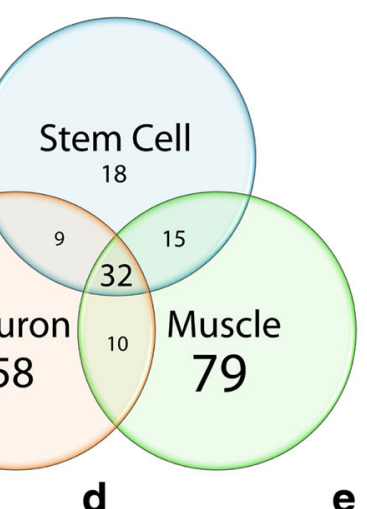

\section{b}
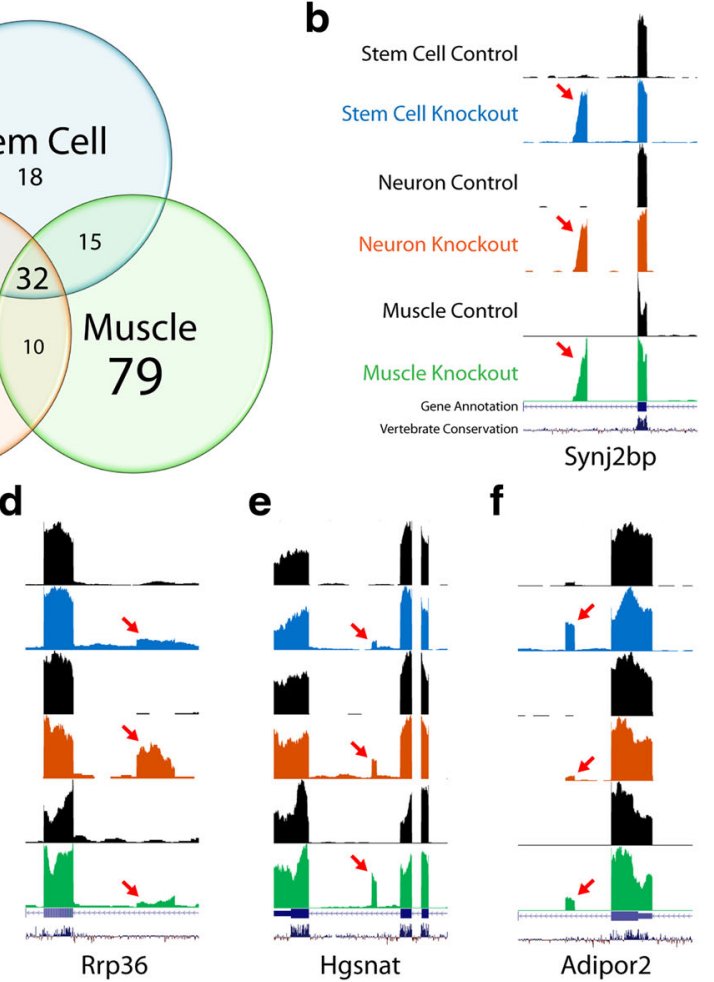

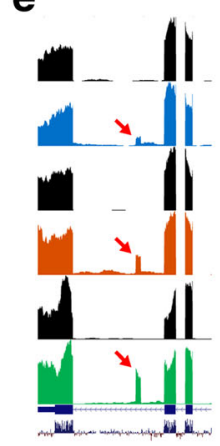

Hgsnat f

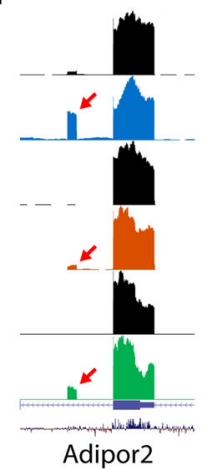

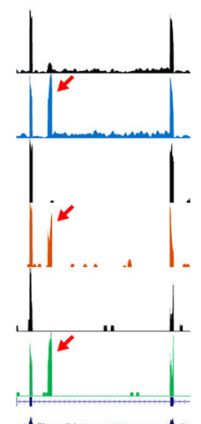

Adnp2

g

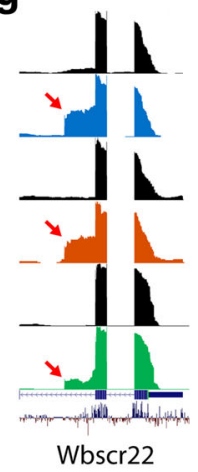

Fig. 4 Tdp-43 cryptic exons are highly variable between cell types. (a) While some cryptic exons are common between cell types, many cryptic exons are unique to neurons (58), muscle (79) and stem cell [22]. Of the common cryptic exons, several are highly incorporated in mRNA regardless of splicing environment (b), while other cryptic exons are incorporated at varying levels depending on the cell type (c to $\mathbf{g}$ )

involved in autophagy [30], and Tbc1d1 and Adipor2 are involved in fat metabolism [20].

Interestingly, a low percentage of cryptic exons ( $6 \%)$ do not induce nonsense mediated decay, but still have an impact on protein structure. These cryptic exons do not contain any stop codons and have sequence lengths that are multiples of three, thereby preventing detrimental frameshifts (Additional file 1: Table S3). These inframe cryptic exons introduce short peptide insertions into the primary amino acid sequence of the protein, which may represent neoantigens.

\section{Discussion}

We have found that Tdp-43's nonconserved cryptic exons vary widely between cell types and affect many pathways that are critical for neuronal and muscle physiology. This suggests that in human disease, myogenic and neurogenic TDP-43 proteinopathies exhibit cell type-specific cryptic exons that could influence disease progression in unique ways. Although our RNA-seq data are based on a limited number of samples, future analysis to increase sample sizes would strengthen our findings. Identifying the cryptic exons that are specific to human neurons or myocytes will also help clarify the selective vulnerability associated with diseases such as IBM and ALS-FTD.

While it remains to be proven whether TDP-43 loss of function is a central driver of human disease, our data demonstrates that within neurons and myocytes, TDP43 is the major splicing repressor for numerous nonconserved cryptic exons. In human disease, dysregulation of Tdp-43 function may impair other neuronal functions beyond mRNA splicing such as axonal trafficking, hyperexcitability, and liquid-liquid phase separation [31-34]. Nevertheless, mouse models of Tdp-43 have demonstrated that constitutive deletion of Tardbp results in embryonic lethality $[24,25,35,36]$. Conditional depletion of Tardbp in adult mice also leads to metabolic deficits and premature death [20] and significant neurodegeneration $[26,37,38]$. Together, these studies demonstrate the importance of Tdp-43 for cell survival.

The current work clarifies the mechanisms of toxicity that underlie Tdp-43 loss of function in the context of cryptic exon repression [15], a finding that has been replicated by other groups [39-41]. Our results suggest that cryptic exons disrupt unique pathways depending on cellular context, although future studies are needed to understand the degree to which these splicing errors 
Table 1 Common pathways affected by Tdp-43 cryptic exons across mouse stem cell, muscle and neuron (cryptic exon present in at least two cell-types)

\begin{tabular}{|c|c|c|c|c|}
\hline $\begin{array}{l}\text { Gene } \\
\text { Symbol }\end{array}$ & Gene Name & \begin{tabular}{|l}
$\overline{\bar{\Phi}}$ \\
$\mathbf{U}$ \\
$\mathrm{E}$ \\
$\mathbf{\Phi}$ \\
$\bar{\omega}$ \\
\end{tabular} & 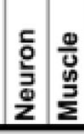 & Keywords \\
\hline \multicolumn{5}{|c|}{ Mitochondria } \\
\hline Synj2bp & Synaptojanin 2 Binding Protein & & & Mitochondrial outer membrane \\
\hline Ptcd2 & Pentatricopeptide Repeat Domain 2 & & & Mitochondrial RNA metabolism \\
\hline Pycr2 & Pyrroline-5-Carboxylate Reductase 2 & & & Proline biosynthesis \\
\hline Cluh & Clustered Mitochondria (CluA/CLU1) Homolog & & & Mitochondrial distribution and biogenesis \\
\hline Letm1 & Leucine Zipper-EF-Hand Containing Transmembrane Protein 1 & & & Mitochondria tubule and cristae organization \\
\hline Mrps6 & Mitochondrial Ribosomal Protein S6 & & & Mitochondrial ribosomal protein \\
\hline \multicolumn{5}{|c|}{ Transcription } \\
\hline$\overline{\text { Adnp2 }}$ & ADNP Homeobox 2 & & & Possible transcriptional regulator \\
\hline Crem & CAMP Responsive Element Modulator & & & Binds to CAMP response element \\
\hline Mier1 & Mesoderm Induction Early Response 1, Transcriptional Regulator & & & Transcriptional repressor \\
\hline Gtt2e2 & General Transcription Factor IIE, Polypeptide 2, Beta 34kDa & & & Binds transcription initiation complex \\
\hline \multicolumn{5}{|c|}{ Genome Regulation and Stability } \\
\hline Hdac4 & Histone Deacetylase 4 & & & Histone deacetylase \\
\hline Wbscr22 & Williams Beuren Syndrome Chromosome Region 22 & & & DNA methyltransferase \\
\hline Brms1l & Breast Cancer Metastasis-Suppressor 1-Like & & & Histone deacetylase complex \\
\hline Chd1I & Chromodomain Helicase DNA Binding Protein 1-Like & & & Helicase; DNA repair \\
\hline Brd9 & Bromodomain Containing 9 & & & Possible chromatin remodeler \\
\hline Ssbp2 & Single-Stranded DNA Binding Protein 2 & & & Genome stability \\
\hline \multicolumn{5}{|c|}{ Ubiquitination } \\
\hline Usp15 & Ubiquitin Specific Peptidase 15 & & & Ubiquitin specific peptidase; TGF- $\beta$ \\
\hline Ube2d1 & Ubiquitin-Conjugating Enzyme E2D 1 & & & E2 ubiquitin ligase \\
\hline Trim8 & Tripartite Motif Containing 8 & & & E3 ubiquitin ligase \\
\hline \multicolumn{5}{|c|}{ Protein and RNA Regulation } \\
\hline Tecpr1 & Tectonin Beta-Propeller Repeat Containing 1 & & & Autophagy \\
\hline Edem2 & ER Degradation Enhancer, Mannosidase Alpha-Like 2 & & & ER-associated misfolded protein degradation \\
\hline Uggt2 & UDP-Glucose Glycoprotein Glucosyltransferase 2 & & & Reglycosylates misfolded glycoproteins \\
\hline Smg5 & SMG5 Nonsense Mediated MRNA Decay Factor & & & Nonsense-mediated decay \\
\hline Hgsnat & Heparan-Alpha-Glucosaminide N-Acetyltransferase & & & Lysosomal acetyltransferase \\
\hline Drosha & Drosha, Ribonuclease Type III & & & miRNA biogenesis \\
\hline \multicolumn{5}{|c|}{ Cell Growth and Homeostasis } \\
\hline Ggct & Gamma-Glutamylcyclotransferase & & & Glutathione homeostasis \\
\hline Adipor2 & Adiponectin Receptor 2 & & & Adiponectin receptor \\
\hline Tbc1d1 & TBC1 (Tre-2/USP6, BUB2, Cdc16) Domain Family, Member 1 & & & Cell cycle; GLUT4-vesicle trafficking \\
\hline Mical2 & Molecule Interacting With CasL Protein 2 & & & Actin depolymerization \\
\hline \multicolumn{5}{|c|}{ Vesicle Trafficking } \\
\hline Vps13d & Vacuolar Protein Sorting 13 Homolog D (S. Cerevisiae) & & & Vacuolar protein trafficking \\
\hline A230046K03Rik & Strumpellin And WASH-Interacting Protein & & & Endosome trafficking \\
\hline Mcoln1 & Mucolipin 1 & & & Vesicular membrane TRP cation channel \\
\hline \multicolumn{5}{|c|}{ Other } \\
\hline Ift81 & Intraflagellar Transport 81 & & & Ciliogenesis \\
\hline Radil & Ras Association And DIL Domains & & & Cell adhesion and migration \\
\hline Pnpla6 & Patatin-Like Phospholipase Domain Containing 6 & & & Phospholipase; neuronal differentiation \\
\hline Rrp36 & Ribosomal RNA Processing 36 Homolog (S. Cerevisiae) & & & 18S rRNA maturation \\
\hline Ppp6c & Protein Phosphatase 6, Catalytic Subunit & & & Protein phosphatase; cell cycle \\
\hline Nme6 & NME/NM23 Nucleoside Diphosphate Kinase 6 & & & Nucleoside triphosphate synthesis \\
\hline & High Incorporation & \multicolumn{3}{|c|}{ Low Incorporation } \\
\hline & Medium Incorporation & \multicolumn{3}{|c|}{ No Incorporation } \\
\hline
\end{tabular}

Refer to Additional file 2 for a full list of cryptic exons

contribute to cell death. Furthermore, TDP-43 belongs to a family of proteins that repress cryptic exons, suggesting that these splicing factors perform a general function in the cell to maintain splicing fidelity [42]. Thus, loss of TDP-43 splicing repression contributes to cell death and the pathways affected by cryptic exon incorporation are likely to be relevant for disease pathogenesis.

The question then becomes, how do we prevent incorporation of nonconserved cryptic exons? Therapeutic 
strategies that aim to directly interfere with cryptic exon splicing (e.g. anti-sense oligonucleotides) will be difficult to envision due to the sizeable number of nonconserved cryptic exons per cell. Furthermore, because nonconserved cryptic exons are different between mouse and human, testing splicing modulators for human cryptic exons in animal models is essentially impossible. However, the general splicing repression function of TDP-43 is conserved. Thus, it may be possible to use mouse models of TDP-43 deletion to specifically test therapeutic strategies that rescue TDP-43 mechanism of action rather than directly targeting individual cryptic exons. One strategy would employ gene therapy to introduce designer splicing factors-chimeric proteins that would couple the UG binding domain of TDP-43 with non-aggregating splicing repressor domains [15] -into neurons or muscles. In principal, this approach would repress most of TDP-43's nonconserved cryptic exons in a manner that would be species-independent.

If neuron loss or skeletal muscle degeneration can be attenuated, such a therapeutic strategy could be rapidly translated into the clinic. Moreover, the observation that cryptic exons can occasionally introduce inframe insertions into mRNA suggests that certain human TDP-43 cryptic exons could represent biomarkers for human disease. We envision the development of specific antibodies to detect neoantigens introduced by human inframe cryptic exons in CSF or blood from patients, serving as either diagnostic biomarkers or tools to monitor the efficacy of treatments in future clinical trials.

\section{Conclusions}

This study demonstrates that Tdp-43 represses a unique set of cryptic exons, depending on cellular context. Thus, the pathways impacted by Tdp-43 loss-of-function and cryptic exon incorporation are likely distinct for each cell type. These results have important implications for human disease, given that Tdp-43 proteinopathy can manifest in various tissues.

\section{Additional files}

Additional file 1: Supplemental figures and tables. (PDF $4449 \mathrm{~kb}$ )

Additional file 2: Cryptic Exon Data Table. (XLSX 59 kb)

\section{Abbreviations}

ALS: Amyotrophic lateral sclerosis; CaMKlla: Calcium/calmodulin-dependent protein kinase II alpha; FTD: Frontotemporal dementia; IBM: Inclusion body myositis; MLC: Myosin light chain 1/3 locus; NMD: Nonsense-mediated decay; TDP-43: Transactive response DNA binding protein $43 \mathrm{kDa}$.

\section{Acknowledgments}

We thank V. Nehus for technical assistance. CamKllaCre and MLC-Cre mice were kindly gifted, respectively, by P. Worley (Johns Hopkins University School of Medicine) and S. Burden (New York University School of Medicine).

\section{Funding}

This work was supported in part by The Robert Packard Center for ALS Research, the Amyotrophic Lateral Sclerosis Association, Target ALS, the JHU Neuropathology Pelda fund, DoD grant W81XWH1110449, Korea Brain Research Institute basic research program Grant No. 2231-415 (to YHJ), the McKnight Memory and Cognitive Disorders Award, and NIH grant R01-NS095969.

\section{Availability of data and materials}

The datasets supporting the conclusions of this article are included within the article and its Additional files 1 and 2. RNA-seq FASTQ sequencing files have been deposited at the NCBI Sequence Read Archive under SRP061340.

\section{Authors' contributions}

All authors designed experiments and interpreted results. JPL performed cryptic exon analyses. YHJ and AND characterized neuron Tdp-43 deletion mice. SZL, KEB and TEL characterized muscle Tdp-43 deletion mice. EM. and BJT assisted with RNA-sequencing. KDL assisted with pathway analysis. JPL and PCW wrote the paper and all authors approved the manuscript.

\section{Authors' information}

Not applicable.

\section{Competing interests}

J.P.L. and P.C.W. have filed a patent application in the United States that refers to the use of cryptic exon incorporation in RNA transcripts identified in human diseases that exhibit TDP-43 proteinopathy as the basis for biomarkers and therapeutic targets/strategies.

\section{Consent for publication}

Not applicable.

Ethical approval and consent to participate Not applicable.

\section{Author details}

1Departments of Pathology, Johns Hopkins University School of Medicine, Baltimore, MD 21205, USA. ²Departments of Neuroscience, Johns Hopkins University School of Medicine, Baltimore, MD 21205, USA. ${ }^{3}$ Departments of Neurology, Johns Hopkins University School of Medicine, Baltimore, MD 21205, USA. ${ }^{4}$ Laboratory of Neurogenetics, NIA, NIH, Bethesda, MD 20892, USA. ${ }^{5}$ Neural Development and Disease Department, Korea Brain Research Institute, Daegu 701-300, South Korea. ${ }^{6}$ Present address: Institute of Psychological Medicine and Clinical Neurosciences, Cardiff University School of Medicine, Cardiff CF24 4HQ, UK.

Received: 25 May 2016 Accepted: 20 December 2016

Published online: 02 February 2017

References

1. Renton $A E$, et al. A hexanucleotide repeat expansion in C9ORF72 is the cause of chromosome 9p21-linked ALS-FTD. Neuron. 2011;72(2):257-68.

2. DeJesus-Hernandez M, et al. Expanded GGGGCC hexanucleotide repeat in noncoding region of C9ORF72 causes chromosome 9p-linked FID and ALS. Neuron. 2011;72(2):245-56

3. Freischmidt A, et al. Haploinsufficiency of TBK1 causes familial ALS and fronto-temporal dementia. Nat Neurosci. 2015;18(5):631-6.

4. Cirulli ET, et al. Exome sequencing in amyotrophic lateral sclerosis identifies risk genes and pathways. Science. 2015;347(6229):1436-41.

5. Ling S-CC, Polymenidou M, Cleveland DW. Converging mechanisms in ALS and FTD: disrupted RNA and protein homeostasis. Neuron. 2013;79(3):416-38.

6. Neumann $M$, et al. Ubiquitinated TDP-43 in frontotemporal lobar degeneration and amyotrophic lateral sclerosis. Science. 2006;314(5796):130-3.

7. Josephs KA, et al. Staging TDP-43 pathology in Alzheimer's disease. Acta Neuropathol. 2014:127:441-50.

8. Josephs KA, et al. Updated TDP-43 in Alzheimer's disease staging scheme. Acta Neuropathol. 2016;131(4):571-85.

9. Watts GDJ, et al. Inclusion body myopathy associated with Paget disease of bone and frontotemporal dementia is caused by mutant valosin-containing protein. Nat Genet. 2004;36(4):377-81. 
10. Weihl CC, et al. TDP-43 accumulation in inclusion body myopathy muscle suggests a common pathogenic mechanism with frontotemporal dementia. J Neurol Neurosurg Psychiatry. 2008;79:1186-9.

11. Hiniker A, Daniels BH, Margeta M. T-Cell-Mediated Inflammatory Myopathies in HIV-Positive Individuals: A Histologic Study of 19 Cases. J Neuropatho Exp Neurol. 2016;75(3):239-45.

12. LaClair KD, et al. Depletion of TDP-43 decreases fibril and plaque $\beta$-amyloid and exacerbates neurodegeneration in an Alzheimer's mouse model. Acta Neuropathol. 2016;132(6):859-73.

13. Lloyd TE. Novel therapeutic approaches for inclusion body myositis. Curr Opin Rheumatol. 2010;22(6):658-64.

14. Lloyd TE, et al. Evaluation and construction of diagnostic criteria for inclusion body myositis. Neurology. 2014;83(5):426-33.

15. Ling JP, Pletnikova O, Troncoso JC, Wong PC. TDP-43 repression of nonconserved cryptic exons is compromised in ALS-FTD. Science. 2015; 349(6248):650-5.

16. Kim D, Langmead B, Salzberg SL. HISAT: a fast spliced aligner with low memory requirements. Nat Methods. 2015;12(4):357-60.

17. Trapnell C, et al. Differential gene and transcript expression analysis of RNA-seq experiments with TopHat and Cufflinks. Nat Protoc. 2012;7:562-78.

18. James Kent W, et al. The human genome browser at UCSC. Genome Res. 2002;12:996-1006.

19. Dennis G, et al. DAVID: Database for Annotation, Visualization, and Integrated Discovery. Genome Biol. 2003;4:P3.

20. Chiang P-M, et al. Deletion of TDP-43 down-regulates Tbc1d1, a gene linked to obesity, and alters body fat metabolism. Proc Natl Acad Sci U S A. 2010;107(37):16320-4.

21. Casanova E, et al. A CamKllalpha iCre BAC allows brain-specific gene inactivation. Genesis. 2001;31(1):37-42.

22. Mourkioti F, Slonimsky E, Huth M, Berno V, Rosenthal N. Analysis of CRE-mediated recombination driven by myosin light chain $1 / 3$ regulatory elements in embryonic and adult skeletal muscle: a tool to study fiber specification. Genesis. 2008;46(8):424-30.

23. Polymenidou $\mathrm{M}$, et al. Long pre-mRNA depletion and RNA missplicing contribute to neuronal vulnerability from loss of TDP-43. Nat Neurosci. 2011;14(4):459-68.

24. Sephton CF, et al. TDP-43 is a developmentally regulated protein essential for early embryonic development. J Biol Chem. 2010;285(9):6826-34.

25. Kraemer BC, et al. Loss of Murine TDP-43 disrupts motor function and plays an essential role in embryogenesis. Acta Neuropathol. 2010;119(4):409-19.

26. Yang C, et al. Partial loss of TDP-43 function causes phenotypes of amyotrophic lateral sclerosis. Proc Natl Acad Sci U S A. 2014;111(12):E1121-9.

27. Feiguin F, et al. Depletion of TDP-43 affects Drosophila motoneurons terminal synapsis and locomotive behavior. FEBS Lett. 2009;583(10):1586-92.

28. Schmid B, et al. Loss of ALS-associated TDP-43 in zebrafish causes muscle degeneration, vascular dysfunction, and reduced motor neuron axon outgrowth. Proc Natl Acad Sci U S A. 2013;110:4986-91.

29. Kawahara Y, Mieda-Sato A. TDP-43 promotes microRNA biogenesis as a component of the Drosha and Dicer complexes. Proc Natl Acad Sci U S A. 2012:109(9):3347-52.

30. Bose JK, Huang C-C, Shen C-KJ. Regulation of autophagy by neuropathological protein TDP-43. J Biol Chem. 2011:286(52):44441-8.

31. Alami NH, et al. Axonal transport of TDP-43 mRNA granules is impaired by ALS-causing mutations. Neuron. 2014;81(3):536-43.

32. Zhang $W$, et al. Hyperactive somatostatin interneurons contribute to excitotoxicity in neurodegenerative disorders. Nat Neurosci. 2016;19(4):2-6.

33. Wegorzewska I, Bell S, Cairns NJ, Miller TM, Baloh RH. TDP-43 mutant transgenic mice develop features of ALS and frontotemporal lobar degeneration. Proc Natl Acad Sci U S A. 2009;106(44):18809-14.

34. Taylor JP, Brown RH, Cleveland DW. Decoding ALS: from genes to mechanism. Nature. 2016;539(7628):197-206.

35. Wu L-S, et al. TDP-43, a neuro-pathosignature factor, is essential for early mouse embryogenesis. Genesis. 2010;48(1):56-62.

36. Tsao W, et al. Rodent models of TDP-43: recent advances. Brain Res. 2012;1462:26-39.

37. Schwenk BM, et al. TDP-43 loss of function inhibits endosomal trafficking and alters trophic signaling in neurons. EMBO J. 2016:35(21):2350-70.

38. Walker AK, et al. Functional recovery in new mouse models of ALS/FTLD after clearance of pathological cytoplasmic TDP-43. Acta Neuropathol. 2015;130(5):643-60.

39. Tan Q, et al. Extensive cryptic splicing upon loss of RBM17 and TDP43 in neurodegeneration models. Hum Mol Genet. 2016. doi:10.1093/hmg/ddw337.
40. Humphrey J, Emmett W, Fratta Pi, Isaacs AM, Plagnol V. Quantitative analysis of cryptic splicing associated with TDP-43 depletion. bioRxiv: 2016;1-21. https://doi.org/10.1101/076117

41. Li Z, Vuong JK, Zhang M, Stork C, Zheng S. Inhibition of nonsense-mediated RNA decay by ER stress. RNA. 2016. doi:10.1261/rna.058040.116.

42. Ling JP, et al. PTBP1 and PTBP2 Repress Nonconserved Cryptic Exons. Cell Rep. 2016;17(1):104-13.

\section{Submit your next manuscript to BioMed Central and we will help you at every step:}

- We accept pre-submission inquiries

- Our selector tool helps you to find the most relevant journal

- We provide round the clock customer support

- Convenient online submission

- Thorough peer review

- Inclusion in PubMed and all major indexing services

- Maximum visibility for your research

Submit your manuscript at www.biomedcentral.com/submit
) Biomed Central 